\title{
Mobility of Spin Probes in Micelles of Cationic Gemini Surfactants Having Dodecyl Groups
}

\section{Tae-Soo Choi, Hiroshi Ichikawa, Jiahe Qian, Yoshio Shimizu, Hirofusa Shirai and Kunihiro Hamada*}

Faculty of Textile Science and Technology, Shinshu University, 3-15-1 Tokida, Ueda, Nagano 386-8567, Japan

\begin{abstract}
The mobility of nonionic spin probes in gemini surfactant (alkanediyl- $\alpha, \omega$-bis (dimethyldodecylammonium bromide)) micelles was investigated by means of electron spin resonance (ESR) measurements to compare with that of conventional surfactant (dodecyltrimethylammonium bromide and ethyldodecyldimethylammonium bromide) micelles. The rotational correlation times, $\tau_{\mathrm{R}}$, of nitroxide spin probes in aqueous surfactant solutions were calculated from ESR spectra and plotted against the surfactant concentrations. As a result, $\tau_{\mathrm{R}}$ abruptly increased in a concentration region, which is believed to correspond with critical micelle concentration (CMC).CMC's determined by means of the spin probe technique were almost the same as those estimated by the electrical conductance measurements. For the conventional surfactants, the $\tau_{\mathrm{R}}$ values became constant below and above $\mathrm{CMC}$, whereas for one of the gemini surfactants they continued to increase above $\mathrm{CMC}$. Furthermore, activation energies for rotation, $E_{\mathrm{a}}{ }^{\mathrm{R}}$, were determined from the temperature dependence of $\tau_{\mathrm{R}} . E_{\mathrm{a}}{ }^{\mathrm{R}}$ was dependent on the kind of the surfactants, suggesting that the mobility of the spin probes changes significantly with micelle structure. Thus the mobility of the spin probes reflects the discrepancy in the microenvironments between the gemini and conventional surfactant micelles.
\end{abstract}

\section{Introduction}

Recently various chemical compounds have been applied as dyeing auxiliaries. For examples, dialkyldimethylammonium bromide containing two tail groups was used for disperse dyeing of polyester, nylon and acetate [1-5]. As a result, the possibility of low temperature dyeing is demonstrated. We have investigated the effects of bolaform electrolytes on the binding of acid dyes with poly (vinylpyrrolidone) [6-9] and the sorption of acid dyes by a nylon 6 film and a silk fiber $[10,11]$. These studies suggest that the bolaform electrolytes can be used as binding controllers or auxiliaries.

On the other hand, gemini surfactants are made up of two identical amphiphilic moieties connected at the level of the head groups or very close to head groups by a spacer group [12,13], which are also referred to as dimeric or bipolar surfactants $[14,15]$. Although the properties of the gemini surfactant micelles have been extensively studied by Zana et al. [16-24], the gemini surfactants have not been applied as dyeing auxiliaries. To use the surfactants as dyeing auxiliaries, it is necessary to get an information about the microenvironments of the gemini surfactant micelles and the behavior of small molecules in them.

In the present study, the mobility of nitroxide spin probes in propanediyl- $\alpha, \omega$-bis (dimethyldodecylammonium bromide) and hexanediyl $-\alpha, \omega$-bis (dimethyldodecylammonium bromide) micelles was investigated by means of electron spin resonance (ESR) measurements to compare with that for the micelles of the 
conventional surfactants, dodecyltrimethylammonium bromide and ethyldodecyldimethylammonium bromide. The rotational correlation times, $\tau_{\mathrm{R}}$, of the spin probes were calculated from ESR spectra, and their surfactant concentration and temperature dependence were investigated. From these results, the difference between the gemini and conventional surfactants in the microenvironments is discussed.

\section{Experimental}

\subsection{Materials}

Two gemini surfactants and two conventional surfactants shown in Fig. 1 were used. Propanediyl- $\alpha$, $\omega$-bis (dimethyldodecylammonium bromide) (DC3-12) and hexanediyl- $\alpha, \omega$-bis (dimethyldodecylammonium bromide) (DC6-12) were synthesized as follows. A mixed solution of n-dodecyl bromide and the corresponding amine $\left(N, N, N^{\prime}, N^{\prime}\right.$-tetramethyl-1,3diaminopropane or $N, N, N^{\prime}, N^{\prime}$-tetramethyl-1,6-di. aminohexane) in nitroethane was stirred at room temperature for 5 days. The products were then filtered, purified by repeated precipitation from ethanol

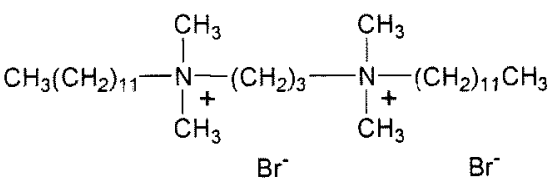

DC3-12

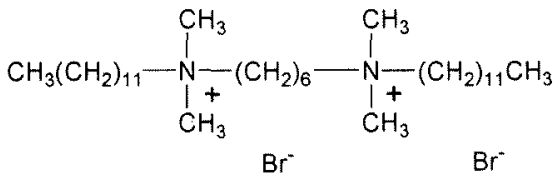

DC6-12<smiles>CCCCCCCC</smiles>
$\mathrm{Br}^{-}$ C $12 \mathrm{C} 1 \mathrm{NBr}$<smiles>CCCCCCCC</smiles>
$\mathrm{C} 12 \mathrm{C} 2 \mathrm{NBr}$

Fig. 1 Surfactants used. into diethylether, and dried. The purity was confirmed by elemental analysis. (Calculated for DC3-12: C, 59.22; $\mathrm{H}, 10.90 ; \mathrm{N}, 4.46 ; \mathrm{Br}, 25.4 \%$. Found: C, 57.76; H, 11.04; $\mathrm{N}, 4.38 ; \mathrm{Br}, 27.0 \%$. Calculated for DC6-12: C, 60.88, H, $11.12, \mathrm{~N}, 4.18 ; 23.8 \%$. Found: C, $60.36 ; \mathrm{H}, 11.54 ; \mathrm{N}, 4.08$; $\mathrm{Br}, 24.7 \%$.)

Dodecyltrimethylammonium bromide $(\mathrm{C} 12 \mathrm{C} 1 \mathrm{NBr})$ was purchased from Tokyo Kasei Kogyo Co. (Tokyo, Japan) and used as received. Ethyldodecyldimethylammonium bromide $(\mathrm{C} 12 \mathrm{C} 2 \mathrm{NBr})$ was synthesized as follows. A mixed solution of $N, N$. dimethyldodecylamine and ethyl bromide in nitroethane was stirred at room temperature for 3 days. After the solvent was removed by evaporation, the product was purified by repeated recrystallization from benzene, treated in diethylether for 1 day, and dried. The purity was confirmed by elemental analysis. (Calculated for $\mathrm{C} 12 \mathrm{C} 2 \mathrm{NBr}$ : C, 59.61; H, 11.26; N, 4.34; Br, $24.8 \%$. Found: C, $59.30 ; \mathrm{H}, 11.18 ; \mathrm{N}, 4.34 ; \mathrm{Br}, 24.7 \%$ )

Two spin probes shown in Fig. 2 were used. The preparation of 2,2,4,4-Tetramethyl-1,2,3,4-tetrahydro$\gamma$-carboline (TTC) was described in a previous paper [25]. 4-Hydroxy-TEMPO benzoate (HTB) was purchased from Aldrich and used as received.

\subsection{Method}

The electric conductivity of aqueous surfactant solutions of various concentrations was measured using a TOA CM-30S conductimeter at $298 \mathrm{~K}$.

The ESR spectra of the aqueous solutions of various surfactant concentrations and a constant spin probe

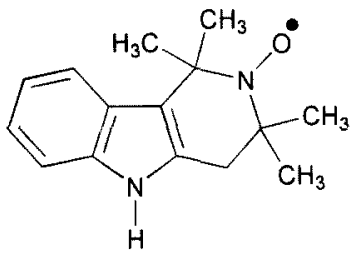

TTC

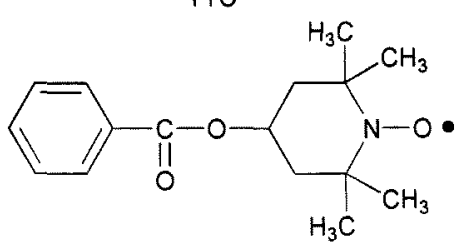

HTB

Fig. 2 Spin probes used. 
concentration $\left(5 \times 10^{-5}\right.$ and $1 \times 10^{-4} \mathrm{~mol} \mathrm{dm}^{-3}$ for TTC and HTB, respectively) were obtained using a JEOL TE-200 ESR spectrometer in the X band at $298 \mathrm{~K}$. The modulation width and the microwave power were kept at $0.05 \mathrm{mT}$ and $1 \mathrm{~mW}$, respectively, to avoid line broadening and saturation effects. For the aqueous solutions of a constant surfactant concentration above CMC $\left(5 \times 10^{-2}\right.$ and $1 \times 10^{-1} \mathrm{~mol} \mathrm{dm}^{-3}$ for $\mathrm{C} 12 \mathrm{ClNBr}$ and $\mathrm{C} 12 \mathrm{C} 2 \mathrm{NBr} ; 5 \times 10^{-3}$ and $1 \times 10^{-1} \mathrm{~mol} \mathrm{dm}^{-3}$ for DC3-12 and DC6-12) and a constant spin probe concentration, the ESR spectra were measured in the temperature range from 273 to $318 \mathrm{~K}$ using a JEOL ES.DVT2 variable temperature controller.

\section{Results and Discussion}

\subsection{Electric Conductivity}

The plots of specific conductance against surfactant concentration at $298 \mathrm{~K}$ are shown in Fig. 3. In all the plots, distinct crossover points were found, which are believed to correspond with the $\mathrm{CMC}$ values of the surfactants. They were $9.41 \times 10^{-4}, 1.20 \times 10^{-3}, 1.52 \times$ $10^{-2}$, and $1.44 \times 10^{-2} \mathrm{~mol} \mathrm{dm}^{-3}$ for DC3-12, DC6-12, $\mathrm{C} 12 \mathrm{C} 1 \mathrm{NBr}$, and $\mathrm{C} 12 \mathrm{C} 2 \mathrm{NBr}$, respectively. These values are consistent with those reported in the literature $[16,26]$. The CMC's of the gemini surfactants are decreased about one order of magnitude under those of the conventional surfactants. This effect is due to the connection of two ammonium head groups.

\subsection{Spin Probes}

The ESR spectra of TTC spin probes are shown in Fig. 4. The peaks in the higher magnetic field measured for the gemini surfactant solutions around $\mathrm{CMC}$ were distinctly split up into two peaks, which are assigned to the peaks for the spin probes in bulk water (peak A) and in the micelles (peak B). Such distinct splitting was not observed in the case of $\mathrm{HTB}$ spin probe. This result is explained by the exchange rate of the spin probe between bulk water and the micelles: the slower exchange rate makes the splitting more distinct. The less distinct splitting for the conventional surfactant solutions around $\mathrm{CMC}$ leads to the fact that the exchange is slower for the gemini surfactants than for the conventional ones. The linkage chains of the gemini surfactants probably prevent the spin probe from exchanging between bulk water and the micelles.

It is convenient to use the area of the peaks $A$ and (a)

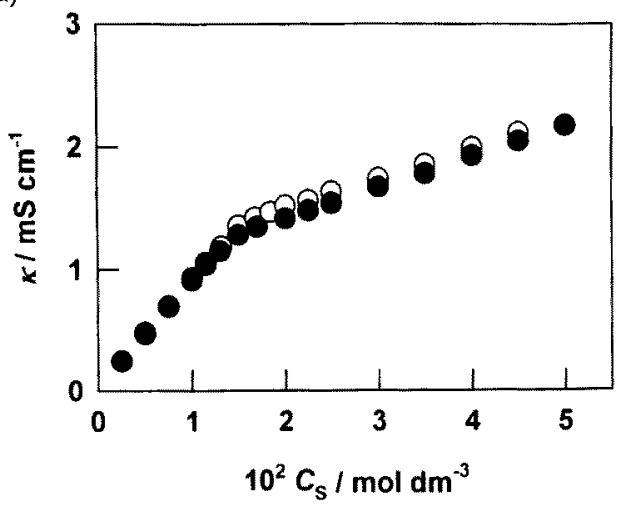

(b)

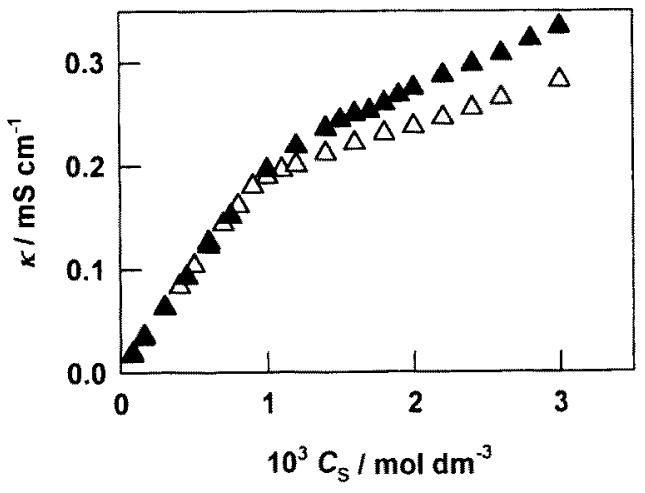

Fig. 3 Dependence of specific conductance on surfactant concentration at $298 \mathrm{~K}$.

(a) $\mathrm{C} 12 \mathrm{C} 1 \mathrm{NBr}(\mathrm{O})$ and $\mathrm{C} 12 \mathrm{C} 2 \mathrm{NBr}(-)$;

(b) $\mathrm{DC} 3-12(\Delta)$ and DC6-12 (A).

$\mathrm{B}$ for determining the binding constants, $K$, of the spin probe, TTC, with the gemini surfactant micelles. Now we assume the following equilibrium:

Probe + Micelle $\rightleftarrows$ Probe/Micelle Complex

Here the probe/micelle complex shows the probe solubilized into the micelle. Considering the probe and micelle concentration, one probe molecule is believed to be solubilized into one micelle. $K$ is expressed by eq 2 .

$$
K=\frac{[\text { Complex }]}{[\text { Probe }] \cdot\left(C_{\text {surf }}-C M C\right) / n}
$$

where [Probe] is the probe concentration in bulk water, $\mathrm{C}_{\text {surf }}$ the surfactant concentration, $n$ the number of the surfactant in a micelle, and [Complex] the complex concentration which equals the concentration of the probe solubilized into the micelle. Here it is assumed that the total micelle concentration is much larger than [Complex]. If the area of the peaks $A$ and $B$ is 
(a) $8 \times 10^{-4} \mathrm{~mol} \mathrm{dm}^{-3}$

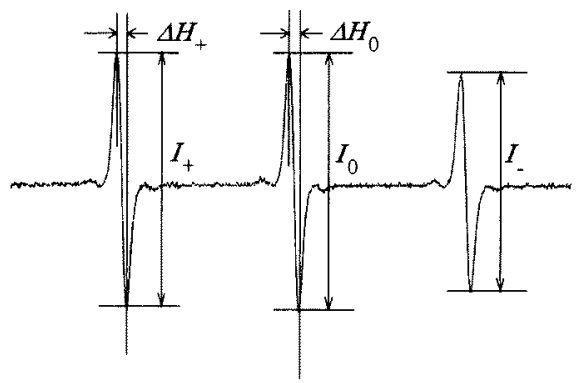

(b) $7 \times 10^{-3} \mathrm{~mol} \mathrm{dm}^{-3}$

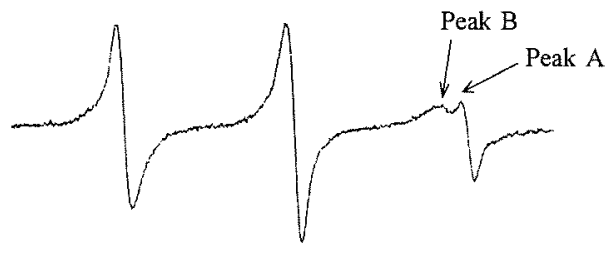

(c) $1 \times 10^{-1} \mathrm{~mol} \mathrm{dm}^{-3}$

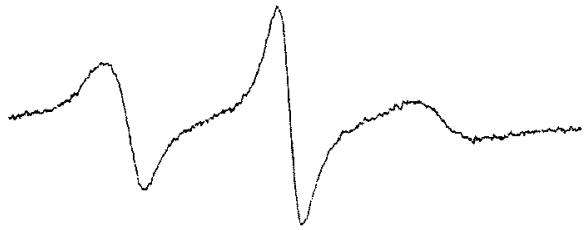

Fig. 4 ESR spectra of TTC in the presence of DC3-12.

designated as $A^{\mathrm{A}}$ and $A^{\mathrm{B}}$, respectively, Eq. 2 can be rewritten by $\mathrm{Eq} .3$.

$$
K / n=\frac{A^{\mathrm{B}}}{A^{\mathrm{A}} \cdot\left(C_{\text {surf }}-C M C\right)}
$$

The $K / n$ values thus obtained are given in Table 1 . Although they have large errors because of the above assumption, $K / n$ is believed to increase with increasing temperature both for DC3-12 and DC6-12. The apparent thermodynamic parameters, the apparent enthalpy change and the apparent entropy change were determined as $8.5 \mathrm{~kJ} \mathrm{~mol}^{-1}$ and $40 \mathrm{~J} \mathrm{~mol}^{-1} \mathrm{~K}^{-1}$ for DC3 12 , and $6.5 \mathrm{~kJ} \mathrm{~mol}^{-1}$ and $40 \mathrm{~J} \mathrm{~mol}^{-1} \mathrm{~K}^{-1}$ for DC6. 12 , respectively, from the temperature dependence of $K / n$. Even if the temperature dependence of the aggregation number, $n$, is taken into consideration to explain the parameters, the binding process of the spin probe witl the gemini surfactant micelles seems to be endothermi and entropic. Furthermore, $K / n$, which means thr binding constant per one gemini surfactant molecule for DC6.12 was larger than that for DC3.12. The fac that the aggregation number, $n$, for DC6-12 is smalles than that for DC3-12 [21] makes it obscure how the binding constant per one micelle, $K$, is influenced by the spacer chain length.

The rotational correlation times, $\tau_{R}$, of the spir probes were determined from the ESR spectra using Eqs. 4, 5, and 6 derived by Kivelson [27].

$$
\begin{aligned}
& \tau_{\mathrm{R}}{ }^{1}=-K_{1} \Delta H_{+}\left(R_{-} / R_{+}-1\right) \\
& \tau_{\mathrm{R}}{ }^{2}=K_{2} \Delta H_{0}\left(R_{+}+R_{-}-2\right) \\
& \tau_{\mathrm{R}}{ }^{3}=K_{1} \Delta H_{0}\left(R_{+}-R_{-}\right)
\end{aligned}
$$

\begin{tabular}{|c|c|c|c|}
\hline Surfactants & $\begin{array}{l}\text { Concentration } \\
\left(\mathrm{mol} \mathrm{dm}{ }^{-3}\right)\end{array}$ & $\begin{array}{c}\text { Temperature } \\
\text { (K) }\end{array}$ & $\begin{array}{c}K / n \\
\left(\mathrm{dm}^{3} \mathrm{~mol}^{-1}\right)\end{array}$ \\
\hline \multirow[t]{13}{*}{ DC3-12 } & $2.5 \times 10^{-3}$ & 298 & 4.23 \\
\hline & $5 \times 10^{-3}$ & 283 & 3.75 \\
\hline & $5 \times 10^{-3}$ & 288 & 3.23 \\
\hline & $5 \times 10^{-3}$ & 293 & 3.60 \\
\hline & $5 \times 10^{-3}$ & 298 & 3.61 \\
\hline & $5 \times 10^{-3}$ & 303 & 3.77 \\
\hline & $5 \times 10^{-3}$ & 308 & 3.70 \\
\hline & $5 \times 10^{-3}$ & 313 & 5.05 \\
\hline & $5 \times 10^{-3}$ & 318 & 5.38 \\
\hline & $7 \times 10^{-3}$ & 298 & 5.04 \\
\hline & $8 \times 10^{-3}$ & 298 & 5.88 \\
\hline & $1 \times 10^{-2}$ & 298 & 5.87 \\
\hline & $2 \times 10^{-2}$ & 298 & 5.13 \\
\hline \multirow[t]{12}{*}{ DC6-12 } & $1.4 \times 10^{-3}$ & 298 & 10.27 \\
\hline & $1.6 \times 10^{-3}$ & 298 & 10.69 \\
\hline & $2 \times 10^{-3}$ & 298 & 7.27 \\
\hline & $3 \times 10^{-3}$ & 283 & 6.23 \\
\hline & $3 \times 10^{-3}$ & 288 & 8.71 \\
\hline & $3 \times 10^{-3}$ & 293 & 8.56 \\
\hline & $3 \times 10^{-3}$ & 298 & 11.45 \\
\hline & $3 \times 10^{-3}$ & 303 & 8.06 \\
\hline & $3 \times 10^{-3}$ & 308 & 8.41 \\
\hline & $3 \times 10^{-3}$ & 313 & 9.10 \\
\hline & $3 \times 10^{-3}$ & 318 & 10.63 \\
\hline & $6 \times 10^{-3}$ & 298 & 21.72 \\
\hline
\end{tabular}

Table 1 Binding Constants of TTC with the Gemini Surfactant Micelles, $K / n$. 
where $R_{ \pm}=\left(I_{0} / I_{+}\right)^{1 / 2}$, and $I_{+}, I_{0}$, and $I$ - are the amplitudes of the peaks in the low, center, and high field, respectively, as shown in Fig. 4. $\Delta H_{+}$and $\Delta H_{0}$ are the peak-to-peak derivative widths in the low and center field, respectively. The constants, $K_{1}$ and $K_{2}$, vary significantly from probe to probe and from system to system for a given spin probe [25]. Here the values described in the literature [25] were used for $K_{1}$ and $K_{2}, \tau_{\mathrm{R}}{ }^{1}, \tau_{\mathrm{R}}{ }^{2}$, and $\tau_{\mathrm{R}}{ }^{3}$ are mathematically equal in the case of the isotropic rotation, while in the case of anisotropic rotation, at least two of these values are different. In all the cases investigated here, three rotational correlation times were almost the same (rotation is isotropic), so that $\tau_{\mathrm{R}}{ }^{2}$ will be used and written as $\tau_{\mathrm{R}}$ in the following discussion.

The change of $\tau_{\mathrm{R}}$ with the surfactant concentration, $C_{\text {surr, }}$ is shown in Figs. 5 and 6 . The manner of the change for $\mathrm{C} 12 \mathrm{C} 2 \mathrm{NBr}$ is similar to that for $\mathrm{C} 12 \mathrm{C} 1 \mathrm{NBr}$ : $\tau_{\mathrm{R}}$ abruptly increased in a narrow concentration region and it was constant above and below the concentration region. The concentration where $\tau_{\mathrm{R}}$ begins to rise abruptly is thought to be CMC. On the other hand, the rotational correlation times for the gemini surfactants increased gradually. Furthermore, $\tau_{\mathrm{R}}$ for DC3-12 continued to increase even in a higher concentration region than $\mathrm{CMC}$, although $\tau_{\mathrm{R}}$ for $\mathrm{DC} 6-12$ became constant in the higher concentration region. This is explained by the change of the DC3-12 micelle structure and the aggregation number: the DC3-12 micelle structure changes with the surfactant concentration,

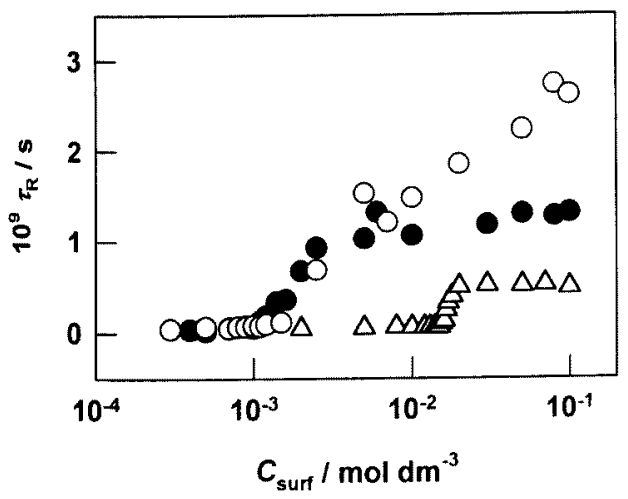

Fig. 5 Dependence of the rotational correlation times, $\tau_{R}$, for TTC on surfactant concentration at $298 \mathrm{~K} \cdot \mathrm{DC} 3-12$ (O), DC6-12 (-), and $\mathrm{C} 12 \mathrm{C} 1 \mathrm{NBr}(\Delta)$.

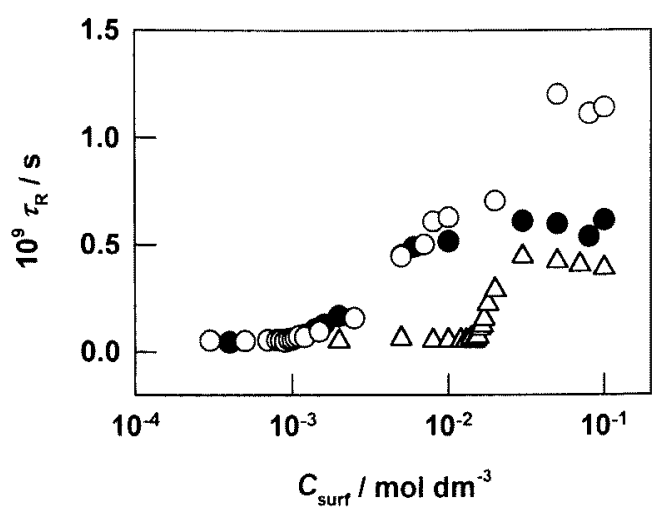

Fig. 6 Dependence of the rotational correlation times, $\tau_{\mathrm{R}}$, for $\mathrm{HTB}$ on surfactant concentration at $298 \mathrm{~K}$. DC3-12 (O), DC6-12 (O), and $\mathrm{Cl} 2 \mathrm{C} 1 \mathrm{NBr}(\Delta)$.

which is reflected by the mobility of the spin probes. The time resolved fluorescence quenching technique also demonstrated that the aggregation numbers for the gemini surfactant micelles having the shorter spacer chains were quite dependent on the surfactant concentration [21].

CMC's determined from ESR measurements are given in Table 2. CMC's for the gemini surfactants have large errors because the crossover points are not clear, but they are consistent with those determined by the conductivity measurements.

It is noteworthy that $\tau_{\mathrm{R}}$ in the gemini surfactant micelles is larger than that for the conventional surfactant micelles. This suggests that the rotational motion of the spin probes is more restricted in the gemini surfactant micelles and the inner viscosity is different between the gemini and conventional surfactant micelles. In addition, $\tau_{\mathrm{R}}$ in the gemini surfactant micelles varied from probe to probe (the mobility of HTB was higher than that of TTC), whereas the mobility in the conventional surfactant micelles was almost the same for both the spin probes. This result is presumably explained as follows. As the location of the spin probes in the micelles is dependent on their hydrophobicity, the difference in the mobility between both the spin probes should reflect their location. If the location of two spin probes is different, the similar mobility in the conventional surfactant micelle should be due to the homogeneous viscosity of the micelle core. The presence of the linked alkyl chains 
Table 2 CMC's Determined by Means of Two Methods $\left(\mathrm{mol} \mathrm{dm}^{-3}\right)$ at $298 \mathrm{~K}$

\begin{tabular}{lccc}
\hline \multirow{2}{*}{ Surfactants } & Conductivity & \multicolumn{2}{c}{ Spin probes } \\
\cline { 3 - 4 } & & TTC & HTB \\
\hline C12C1NBr & $1.52 \times 10^{-2}$ & $1.4 \times 10^{-2}$ & $1.5 \times 10^{-2}$ \\
C12C2NBr & $1.44 \times 10^{-2}$ & $1.3 \times 10^{-2}$ & $1.3 \times 10^{-2}$ \\
DC 3.12 & $9.41 \times 10^{-4}$ & $1.2 \times 10^{-3}$ & $1.2 \times 10^{-3}$ \\
DC6.12 & $1.20 \times 10^{-3}$ & $1.1 \times 10^{-3}$ & $1.2 \times 10^{-3}$ \\
\hline
\end{tabular}

probably induces the heterogeneous viscosity of the gemini surfactant micelle core.

For further discussion, the temperature dependence of $\tau_{R}$ was investigated. The Arrhenius plots of $\tau_{R}$ for TTC are shown in Figs. 7 and 8 . The $\tau_{\mathrm{R}}$ values for 0.005 mol dm${ }^{-3}$ aqueous solution of DC3-12 were calculated by separating the ESR peaks in bulk water. In the case of HTB, similar Arrhenius plots were obtained. As described above, $\tau_{\mathrm{R}}$ increased in the order of water $<$ $\mathrm{C} 12 \mathrm{C} 1 \mathrm{NBr}(\mathrm{C} 12 \mathrm{C} 2 \mathrm{NBr})<\mathrm{DC} 6-12<\mathrm{DC} 3-12$.

The activation energies for rotation, $E_{\mathrm{a}}{ }^{\mathrm{a}}$, were calculated from the slopes of the Arrhenius plots and are given in Table 3. $E_{\mathrm{a}}{ }^{\mathrm{R}}$ in the micelles except those for $5 \times 10^{-3} \mathrm{~mol} \mathrm{dm}^{-3}$ aqueous solutions of DC3-12 and DC6.12 was much larger than that in water for both the spin probes. Because in the lower gemini surfactant region where $\tau_{R}$ continues to increase as shown in Fig. 5 , the micelle inner structure is different or premicelle is formed instead of real micelle, $E_{\mathrm{a}}{ }^{\mathrm{R}}$ in the lower gemini

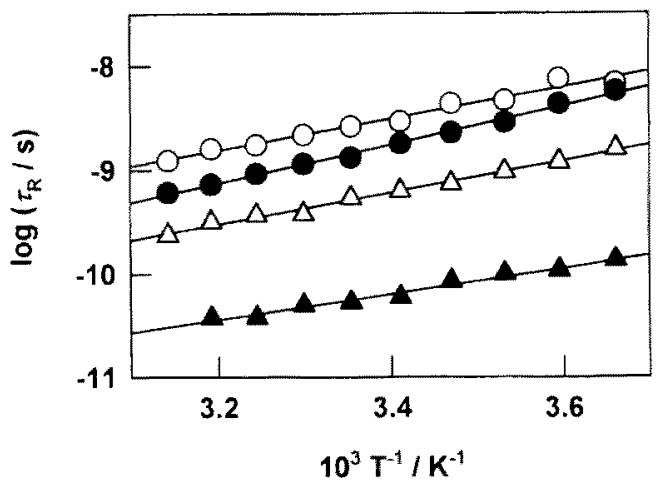

Fig. 7 Arrhenius plots of the rotational correlation times, $\tau_{R}$, for TTC. DC3-12 $\left(1 \times 10^{-1} \mathrm{~mol} \mathrm{dm}^{-3}\right)$ (O), DC6.12 (1 $\left.\times 10^{-1} \mathrm{~mol} \mathrm{dm}^{-3}\right)$ (๑), $\mathrm{C} 12 \mathrm{C} 1 \mathrm{NBr}\left(5 \times 10^{-2} \mathrm{~mol} \mathrm{dm}^{-3}\right)(\Delta)$, and water (A).

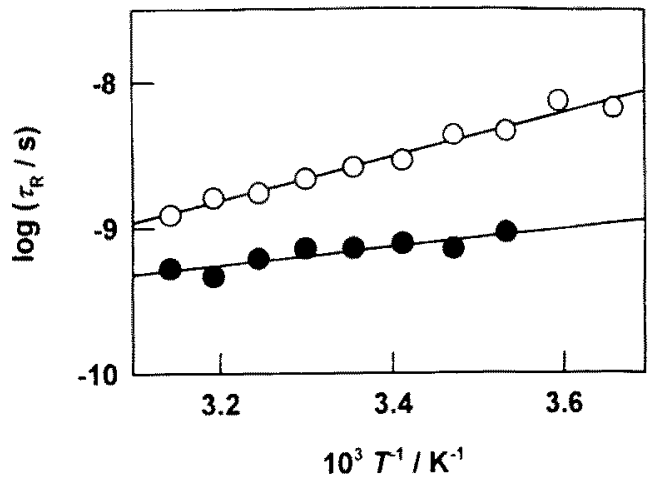

Fig. 8 Arrhenius plots of the rotational correlation times, $\tau_{\mathrm{F}}$, for TTC in DC3-12 micelle. $1 \times 10^{-1}$ $\mathrm{mol} \mathrm{dm} \mathrm{d}^{-3}(\mathrm{O})$ and $5 \times 10^{-3} \mathrm{~mol} \mathrm{dm}^{-3}(\mathbf{O})$.

surfactant concentration region is much smaller than in the higher concentration region. In the following section, $E_{\mathrm{a}}{ }^{\mathrm{R}}$ in higher concentration region is discussed.

$E_{\mathrm{a}}{ }^{\mathrm{R}}$ in the DC3.12 micelle was similar to those in the conventional surfactant micelles, whereas the higher activation energies were found in the DC6-12 micelles. As was pointed out in previous papers [28-30], the mode of rotation should be taken into consideration to interpret the activation energies. If the motion of the spin probes consists of rotation and translation, $E_{\mathbf{a}}{ }^{\mathbf{B}}$ includes a part of the activation energy for translation in addition to that for rotation [30]. This suggests that the rotational mode of the spin probes is probably different between DC3-12 and DC6-12 micelles. In addition, $E_{\mathrm{a}}{ }^{\mathrm{R}}$ for DC6-12 was different between TTC and HTB. This might reflect the location of the spin

Table 3 Activation Energies for Rotation, $E_{\mathrm{a}}{ }^{\mathrm{R}}\left(\mathrm{kJ} \mathrm{mol}^{-1}\right)$

\begin{tabular}{lccc}
\hline Surfactants & $\begin{array}{c}\text { Concentration } \\
\left(\mathrm{mol} \mathrm{dm}^{-3}\right)\end{array}$ & TTC & HTB \\
\hline Water & & $23.7 \pm 1.4$ & $21.4 \pm 1.7$ \\
C12C1NBr & $5 \times 10^{-2}$ & $28.9 \pm 1.0$ & \\
& $1 \times 10^{-1}$ & & $28.6 \pm 1.0$ \\
C12C2NBr & $5 \times 10^{-2}$ & $26.5 \pm 1.3$ & $27.2 \pm 1.0$ \\
& $1 \times 10^{-3}$ & $28.7 \pm 1.5$ & $29.7 \pm 1.0$ \\
DC3-12 & $5 \times 10^{-3}$ & $12 \pm 2$ & \\
& $1 \times 10^{-1}$ & $29.0 \pm 1.7$ & $29.2 \pm 0.6$ \\
DC6-12 & $5 \times 10^{-3}$ & $18 \pm 3$ & \\
& $1 \times 10^{-1}$ & $35.4 \pm 0.9$ & $31.2 \pm 1.0$ \\
& & &
\end{tabular}


probes in DC6-12 micelle, as described in the previous section. The similar activation energies for two spin probes in DC3-12 micelle demonstrate that the rotational mode should be considered: the combination of the mode and the heterogeneous viscosity gives rise to the incidental coincidence because the rotational mode and microviscosity around the spin probes are different between TTC and HTB.

From the above results, it is concluded that the mobility of the spin probes reflects the micelle inner properties and elucidates the discrepancy of the micelle structure between the gemini and conventional surfactants. This suggests that the gemini surfactants behave as dyeing auxiliaries in a different manner from the conventional ones.

\section{Acknowledgement}

This work was supported by Grant-in-Aid for COE Research (10CE2003) by the Ministry of Education, Science, Sports and Culture of Japan.

\section{References}

1. I. S. Kim, K. Kono, and T. Takagishi, Text. Res. J., 66, 763 (1996)

2. I. S. Kim, K. Kono, and T. Takagishi, Text. Res. J., 67, 555 (1997).

3. I. S. Kim, K. Kono, and T. Takagishi, Text. Res. J., 67, 664 (1997)

4. I. S. Kim, K. Kono, and T. Takagishi, Text. Res. J., 67, 767 (1997).

5. I. S. Kim, Y. Ishikawa, K. Kono, and T. Takagishi, Text. Res. J., 68, 422 (1998).

6. J. Qian, K. Hamada, and M. Mitsuishi, Dyes Pigm., 25, 167 (1994).

7. J. Qian, K. Hamada, and M. Mitsuishi, Dyes Pigm., 26, 217 (1994)

8. K. Hamada, J. Qian, Y. Hirata, K. Satomura, and M. Mitsuishi, Dyes Pigm., 31, 19(1996).

9. K. Hamada, K. Satomura, and M. Mitsuishi, Dyes Pigm., 31, 205 (1996).
10. K. Hamada, H. Haizuka, and A. Yamazaki, J. Appl. Polym. Sci., 69, 2247 (1998).

11. K. Hamada, J. Qian, and M. Mitsuishi, J. Soc. Dyers Colour., 115, 136(1999).

12. F. M. Menger and C. A. Littau, J. Am. Chem. Soc., 113, 1451 (1991).

13. F. M. Menger and C. A. Littau, J. Am. Chem. Soc., 115, 10083 (1993).

14. J. H. Fuhrhop and D. Fritsh, Acc. Chem. Res., 19, 130 (1986).

15. R. Zana, and Y. Talmon, Nature, 362, 228(1993).

16. R. Zana, M. Benrraou, and R. Rueff, Langmuir, 7, 1072 (1991).

17. E. Alami, H. Levy, R. Zana, and A. Skoulios, Langmuir, 9, 940 (1993).

18. E. Alami, G. Beinert, P. Marie, and R. Zana, Langmuir, 9, 1465(1993).

19. M. Frindi, B. Michels, H. Levy, and R. Zana, Langmuir, 10, 1140(1994).

20. F. Kern, F. Lequeux, R. Zana, and S. J. Candau, Langmuir, 10, 1714(1994).

21. D. Danino, Y. Talmon, and R. Zana, Langmuir, 11, 1448(1995)

22. D. Danino, Y. Talmon, H. Levy, G. Beinert, and R. Zana, Science, 269, 1420(1995).

23. R. Zana, M. In, H. Levy, and G. Duportail, Langmuir, 13, 5552(1997).

24. R. Zana and H. Levy, Colloids Surfaces $A$ : Physicochem. Eng. Aspects, 127, 229 (1997).

25. R. McGregor, T. Iijima, T. Sakai, R. D. Gilbert, and K. Hamada, J. Membr. Sci., 18, 129 (1984).

26. M. N. Jones and J. Piercy, J. Chem. Soc., Faraday Trans. I, 68, 1839 (1972).

27. D. Kivelson, J. Chem. Phys., 33, 1094 (1960).

28. K. Hamada, T. Iijima, and R. McGregor, Macromolecules, 19, 1443(1986).

29. K. Hamada, T. Iijima, and R. McGregor, Macromolecules, 20, 215(1987).

30. K. Hamada, K. Amachi, K. Yonetake, T. Iijima, and R. McGregor, Polym. J., 19, 701 (1987). 